\title{
Editorial
}

Karl-Heinz Paqué

\section{Gesellschaftliche Herausforderungen, ökonomische Antworten}

DOI 10.1515/pwp-2015-0015

\section{Liebe Leserin, lieber Leser,}

Ziel der Perspektiven der Wirtschaftspolitik (PWP) ist es, über große gesellschaftliche Herausforderungen mit wirtschaftswissenschaftlicher Methodik, aber allgemein verständlich zu diskutieren. Das vor Ihnen liegende Heft 3/15 liefert in dieser Hinsicht ein besonders breites Spektrum an Themen.

Ein Schwerpunkt ist dabei die demographische Entwicklung. In einem Übersichtsartikel behandelt Friedrich Breyer den Zusammenhang zwischen der Alterung der Gesellschaft und den Ausgaben für Gesundheit. Er kommt zu dem Ergebnis, dass eine Zunahme dieser Ausgaben wohl unvermeidlich ist und je nach Art des technischen Fortschritts in der medizinischen Versorgung sogar stärker ausfallen kann, als heute prognostiziert wird. Noch mehr Kostendynamik ist in der Pflegeversicherung zu erwarten, zumal dort das Leistungsniveau bisher eher unzureichend ausfällt und deshalb wohl noch in der Zukunft angehoben werden muss. Gesundheit wird also in der volkswirtschaftlichen Wertschöpfung einen zunehmenden Anteil ausmachen, was drängende Fragen nach der Art der Finanzierung - Zwangsbeiträge oder freiwillige Zahlungen - aufwirft. Ebenfalls um Demographie geht es in dem Beitrag aus der Forschung von Axel Börsch-Supan, Michela Coppola und Johannes Rausch. Konkret: um „die Rente mit 63“, eingeführt im vergangenen Jahr von der deutschen Bundesregierung trotz des Trends zur Alterung der Gesellschaft. Die Autoren schätzen empirisch die Auswirkungen auf die gesetzliche Rentenversicherung und analysieren die Verteilungswirkungen. Das Ergebnis: Viele zahlen in Form niedrigerer Renten für das eingeführte Privileg von Wenigen.

Brisant Politisches untersuchen auch Kerstin Bruckmeier, Georg-Benedikt Fischer und Berthold Wigger: die Einführung von allgemeinen Studiengebühren in Deutsch-

Karl-Heinz Paqué, Otto-von-Guericke-Universität Magdeburg, E-Mail:paque@ovgu.de

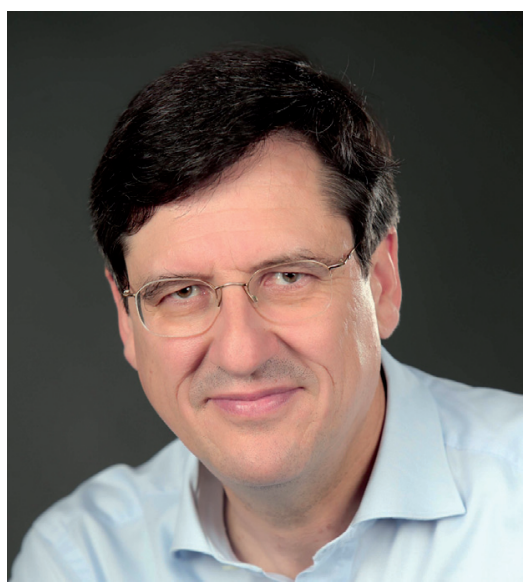

land durch einige Landesregierungen, die allerdings inzwischen Schritt für Schritt zurückgenommen wurde. Mit Blick auf diverse potenzielle Effekte - unter anderem auf Studienneigung, Studienort und Studiendauer sowie öffentliche Ausgaben und sonstige Gebühren - kommen sie zu dem Ergebnis, dass die ermittelten Wirkungen keineswegs eindeutig sind, in jedem Fall aber die Abschaffung der Studiengebühren nicht überzeugend begründen können.

In der Rubrik „Das Gespräch“ - diesmal mit Johann Graf Lambsdorff - steht eine Herausforderung im Vordergrund, die in wohlhabenden Industriegesellschaften oft unterschätzt wird: die Korruption. Die jüngsten Skandale des Fußballweltverbandes FIFA haben allerdings wieder deutlich gemacht, dass es dabei nicht um eine harmlose Randerscheinung geht, die nur Entwicklungsländer betrifft und irgendwann von selbst verschwindet. Lambsdorff gibt Auskunft darüber, ob Korruption zu schweren volkswirtschaftlichen Schäden führt und wie sie modelliert, gemessen und experimentell erforscht werden kann.

Der experimentellen Forschung selbst ist in diesem Heft ein umfangreicher Übersichtsartikel gewidmet. Joachim Weimann resümiert darin die zunehmende Bedeutung der Verhaltensökonomie in der Wirtschaftswissenschaft und der Politikberatung. Es geht dabei auch um zentrale Fragen der Methodik - mit Blick auf die Rationali- 
tät von Entscheidungen sowie die Kausalität, die externe Validität und die Breite der Anwendbarkeit von experimentellen Verfahren. Der Beitrag setzt einen vorläufigen Schlusspunkt zu einer Reihe von Artikeln zu moderner evidenzbasierter Wissenschaft und Politik, die in Heft 2/14 mit einem Blick von Ludger Wößmann (mit Ko-Autoren) auf die Arbeitsweise der modernen Ökonometrie begonnen hatte.

Das vorliegende Heft 3/15 wird wieder einmal von einem Beitrag unserer gelegentlich erscheinenden Rubrik „Aus aktuellem Anlass“ abgeschlossen. Der Autor bin ich selbst, das Thema ist ein neu erschienenes Buch von Barry Eichengreen zum Vergleich der Weltfinanzkrise 2007/08 ff. und der Europäischen Schuldenkrise 2010ff. mit der Weltwirtschaftskrise 1930/32. Das faszinierende Buch dient als Ausgangspunkt für eine Diskussion darüber, inwieweit wir derzeit in der wirtschaftspolitischen Krisendebatte in Europa und den Vereinigten Staaten eine Rückkehr von Ideologien erleben, die dem pragmatischen Lernprozess der Politik nicht ganz gerecht werden.

Karl-Heinz Paqué 